HiLson, G. R. F. (1952). J. gen. Microbiol. 7, 175-178.

\title{
A Simple and Inexpensive Microforge
}

\author{
BY G. R. F. HILSON \\ The Department of Bacteriology, St George's Hospital Medical School, London
}

\begin{abstract}
SUMMARY : A microforge is necessary for fashioning the glass tools used in micromanipulation. A simple method of improvising one from a microscope, an eyecautery and a micromanipulator is described. Its design is based on that of the de Fonbrune microforge. It is capable of the same movements, and the methods of working described for that instrument can be followed.
\end{abstract}

A number of different types of micromanipulator are commercially available, but the glass tools needed for their use are not, and have to be fashioned by means of a microforge for the particular purpose in view. Methods for forging microinstruments have been described clearly and in great detail by de Fonbrune (1949). His descriptions apply to his own microforge, which is a highly versatile instrument, simple to handle and giving reproducible results. Unfortunately it may not be available to every worker needing microtools. The apparatus described here, which was designed to make tools for a Singer high-power micromanipulator (Barer \& Saunders-Singer, 1948), reproduces the main features of the de Fonbrune microforge at a very low cost. Only one part has to be specially made and the machining involved is simple; the rest of the equipment is readily available.

Microforges have been designed by other workers with similar objects in view: cheapness, versatility and reliability. Bretey \& Browaeys (1944) described a simple piece of equipment to be attached to a microscope stage or to one of a pair of micromanipulators. In their instrument the principle of traction by a weight was employed with the pull applied horizontally; the microtool was heated by means of a fine cautery mounted on a micromanipulator. They suggested that all the essential techniques of more expensive microforges could be carried out with their apparatus. A more complicated instrument was described by Thaysen \& Morris (1947); it consisted of a gas forge for preliminary work and an electric forge for the completion of microtools. The tool was mounted on one micronianipulator and the electric forge on another; the principle of traction by weight was not used.

\section{The apparatus and its use.}

The de Fonbrune microforge consists essentially of a low-power microscopc in the horizontal position, fitted with an angle ocular, a rotating mechanical stage, and built-in substage light. The mechanical stage carries a clip which can be turned on an axis perpendicular to the stage, and locked at any point. in its rotation. Thus, by the combined effect of rotating the stage and the clip. a microtool attached to the latter can be set within the optical field at any angle in a vertical plane parallel to the stage. A swivelled mounting on the 
left of the microscope frame carries the heating element and air-blast pipes. The movement of the heating element in the vertical and horizontal planes within the optical field is governed by fine-adjustment controls. The whole forge is fixed on a base which contains the controls which regulate lighting, the strength of the electric current for the heating element, and the strength of the air-blast.

A Zeiss microscope fitted with a rotating mechanical stage and a Beck angle binocular was in use in this laboratory, and it was realized that if a clip capable of carrying a microtool could be fitted to the stage of this instrument,

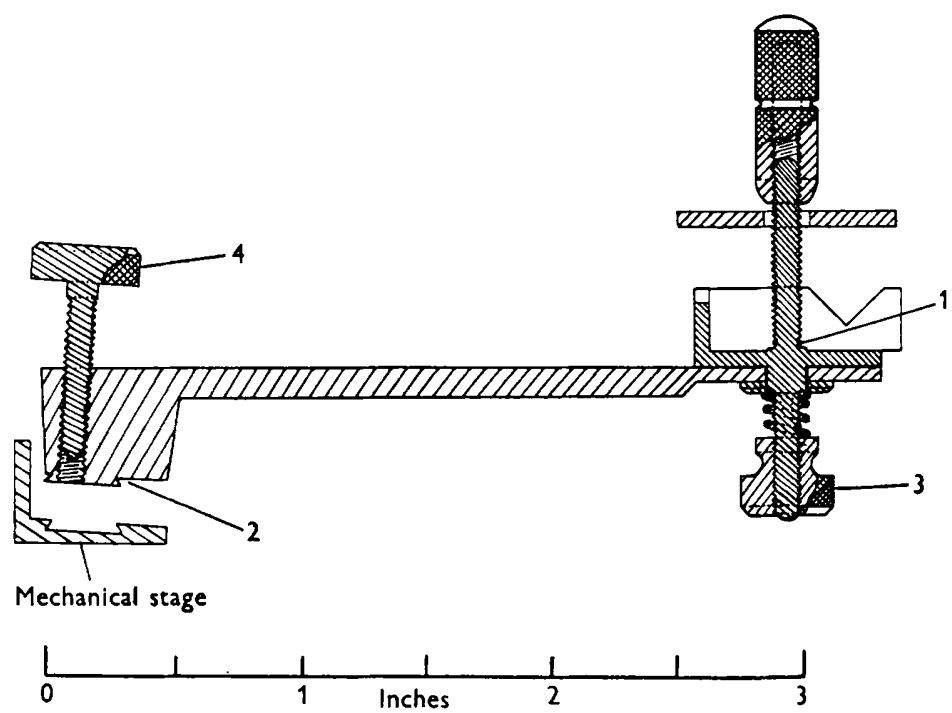

Fig. 1. Sectional drawing of the adapter. A section of the microscope mechanical stage is included to show how the key (2) of the adapter fits into the keyway which normally carries the right-hand clip of the stage. (1) The clip which holds the microtool. (2) The key, which is designed to fit the keyway of the mechanical stage. (3) A spring-loaded nut which controls the rotation of the microtool clip. (4) Locking screw, by means of which the adapter is fixed in position.

an exact functional replica of the optical and mechanical systems of the de Fonbrune machine would be achieved. A simple adapter for this purpose was therefore designed (Fig. 1 and Pl. 1, fig. 1). It consists of a flat strip of plated brass having at one end the clip (1) for the microtool and at the other a key (2) which fits the groove of the mechanical stage. The clip (1) is a modification of the Singer clamp (Registered Design No. 847073). It has a friction bearing with the face of the strip, and is able to rotate completely in one plane with a freedom of movement governed by the tightness of the spring-loaded nut (3). The key (2) is designed to hold the adapter parallel to the microscope stage, and far enough away from it to give clearance between the stage and the nut (3); the adapter is locked into the groove of the stage by the screw (4). This arrangement also has the effect of setting the plane of the microtool sufficiently clear of the mechanical stage to give traction weights an unimpeded 
vertical drop. The adapter is, in fact, nothing but an extension of the mechanical stage made in such a way that it holds the microtool away from the stage and allows it to be rotated at its point of attachment.

The heating element for the microforge is an ordinary surgical eye-cautery mounted in a lightweight cautery handle $(9 \mathrm{~cm}$. long and $1 \mathrm{~cm}$. in diameter). Such handles contain a small switch operated by finger pressure; with the microforge this switch is not used and must be strapped firmly with tape in the 'on' position. The cautery handle is mounted on the micromanipulator to give the fine control needed during the process of forging. Current is provided by a 'cautery box' (output 4 V., 24 amp.) for both the heating element and the substage lamp. Built-in illumination is convenient but may be adequately replaced by an ordinary microscope lamp. The eye-cautery assembly and box are readily available commercially.

The apparatus is set up for use as illustrated (Pl. 1, fig. 2). The microscope is used in the horizontal position; if necessary it may be clipped to a baseboard for greater stability. When the controls for the mechanical stage of the microscope are on the right, the cautery box is placed on that side, and the micromanipulator on the other: for 'left-handed' microscopes this arrangement must be reversed. The adapter is mounted at the right of the mechanical stage and locked into position. The cautery is attached to the micromanipulator so that the cautery handle and the plane of the ' $V$ ' of the heating element are horizontal and level with the optical axis of the microscope. A 1 in. low-power objective is used, and the binocular, fitted with low-power eye-pieces $(\times 6)$, is swung up into the position shown. An angle attachment of this sort, whether monocular or binocular, is very convenient but not indispensable. The rheostats on the cautery box are set to zero and the box is connected to the cautery and substage lamp by their respective flexes.

Preliminary work is carricd out on the microtool by hand as described by de Fonbrune, and it is then clipped on to the adapter. It is adjusted by hand until the tip of the tool appears in the field of view of the microscope when brought into focus. Similarly, the micromanipulator is cautiously moved by hand under naked-eye observation until the tip of the cautery is almost touching the end of the microtool, after which its movements are governed by the controls of the micromanipulator, under vision through the microscope. From this point onwards de Fonbrune's instructions can be followed with little modification.

The design makes no provision for an air-blast. If the microforge is used in conjunction with a Singer micromanipulator, there would be no difficulty in attaching a pipe of suitable shape to the tool rod of this machine, just below the clamp holding the cautery, and connecting it by a flexible lead to a supply of air under pressure. The pipe and cautery would then move together as one unit, an arrangement similar to that found in the de Fonbrune microforge. However, for most of the common microforge techniques an air-blast is not essential.

It should be emphasized that the principal advantage of a microforge of this design is that it is not a permanent piece of equipment for a single purpose 
only. It can be assembled in a minute or two, and, after use, when the adapter has been taken off, the microscope is ready for micromanipulation or for any other use.

My thanks are due to Mr I. M. Macdonald-Murray for carrying out machining and for technical advice.

\section{REFERENCES}

Barer, R. \& Saunders-Singer, A. E. (1948). A new single-control micro-manipulator. Quart. J. micr. Sci. 89, 439.

Bretey, J. \& Browaeys, J. (1944). Confection des instruments au moyen des micromanipulateurs auxquels ils sont destinés. C.R. Soc. Biol., Paris, 138, 465.

de Fonbrune, P. (1949). Technique de micromanipulation. Paris: Masson et Cie.

Thaysen, A. C. \& Morris, A. R. (1947). The preparation of microtools for the micromanipulator. J. gen. Microbiol. 1, 221.

\section{EXPLANATION OF PLATE}

Fig. 1. The mechanical stage with the microscope drawtube removed, showing the adapter in position. A partly fashioned microtool is mounted in the clip of the adapter.

Fig. 2. A general view of the complete assembly. The Singer micromanipulator is on the left, carrying the eye-cautery. The microscope in the centre has a partly processed microtool in the same optical plane as the tip of the cautery, and carries a substage light: at this angle the latter is hidden behind the stage. The cautery box controlling the current to the cautery and substage lamp is on the right.

(Received 27 February 1952) 
Journal of General Microbiolog.y, Vol. 7, Nos. 1 \& 2

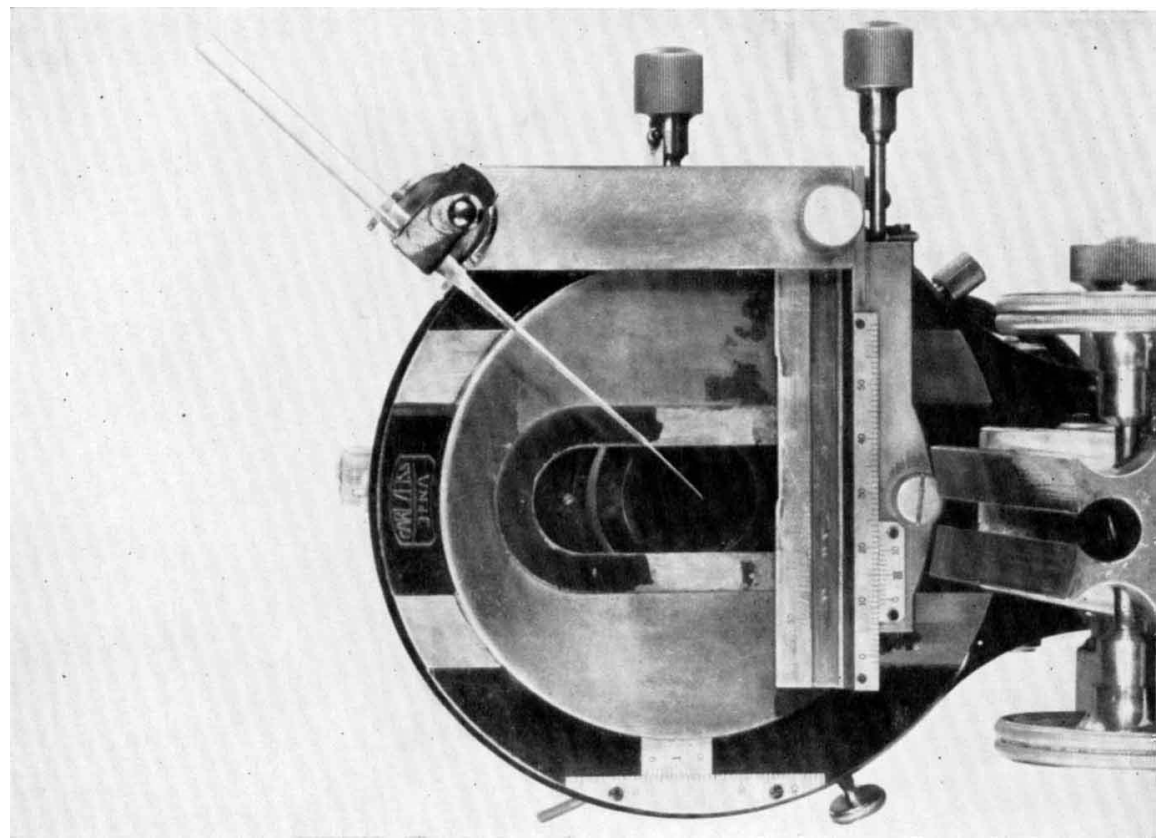

Fig. 1

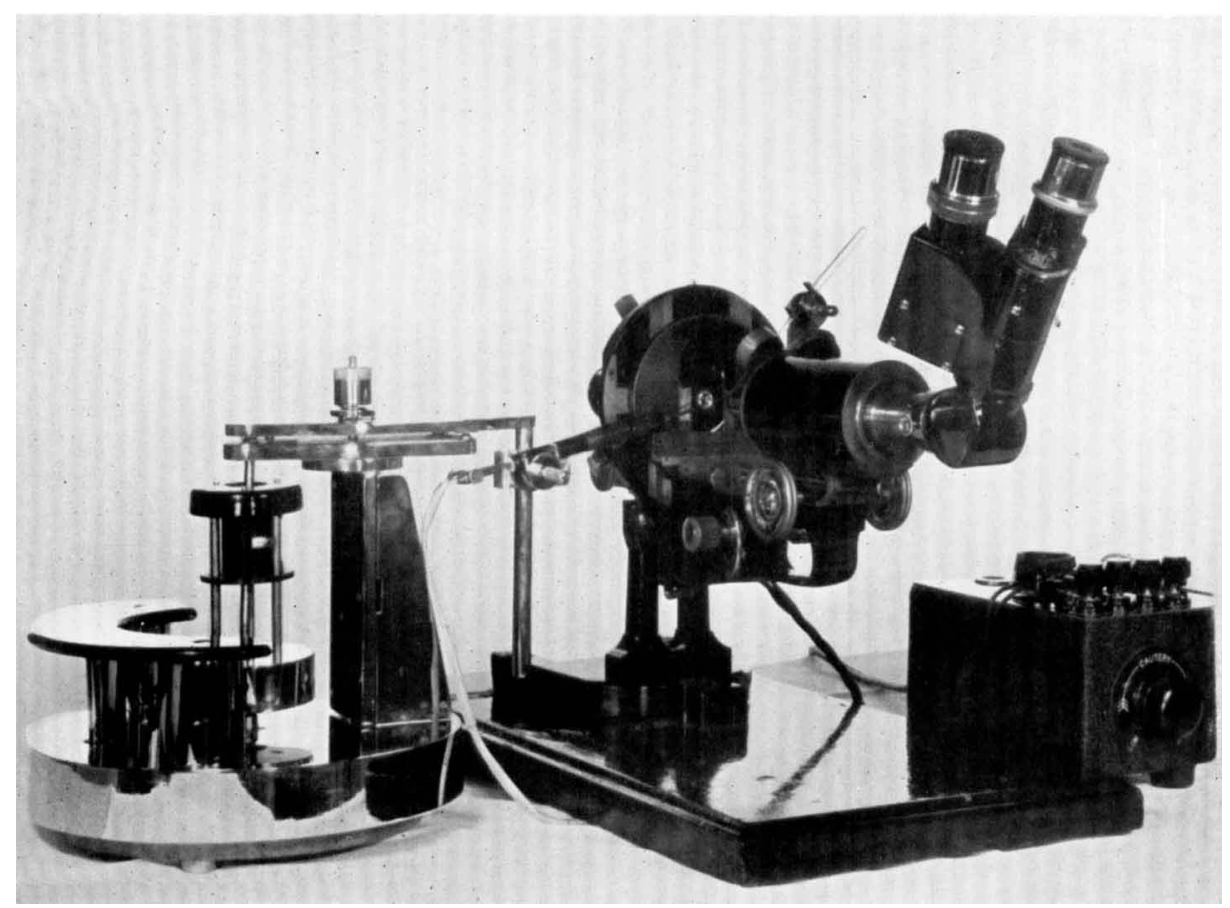

Fin. 2

G. R. F. IILson-A simple microforgh. Plate 1 